\title{
Baromètre BIM : une enquête sur l'adoption du BIM dans les agences d'architecture en France
}

\section{BIM barometer: A questionnaire survey on BIM adoption in French architecture firms}

\author{
Elodie Hochscheid ${ }^{1,2,}$ et Gilles Halin ${ }^{1,3}$ \\ ${ }^{1}$ Laboratoire MAP-CRAI (UMR 3495, CNRS/MC), 2 rue Bastien Lepage, Nancy, France \\ ${ }^{2}$ Ecole Nationale Supérieure d'Architecture de Nancy, 2 rue Bastien Lepage, Nancy, France \\ ${ }^{3}$ Université de Lorraine, Nancy, France
}

\begin{abstract}
Résumé. Le Building Information Modeling (BIM) est un ensemble de technologies et méthodes de travail de plus en plus utilisées et plébiscitées en France et à travers le monde. Il préoccupe les professionnels du secteur de la construction et, en premier lieu, les architectes. Notre connaissance du niveau d'adoption du BIM par les architectes aujourd'hui est pourtant très limitée. Cet article propose d'étudier l'avancement des agences d'architecture dans leur adoption du BIM, et les caractéristiques de celles qui mettent en œuvre ces pratiques.
\end{abstract}

Mots-clés. BIM, architectes, agences d'architecture, questionnaire.

\begin{abstract}
Building Information Modeling (BIM) is a set of technologies and methods which are increasingly being used in France and worldwide. BIM concerns the construction industry stakeholders, especially architects. However, our knowledge of architects' BIM adoption is sketchy. In this paper, it is proposed to identify the progress of architecture firms in BIM adoption, and the characteristics of firms that have implemented BIM.
\end{abstract}

Keywords. BIM, architects, architecture firms, questionnaire survey.

\section{Introduction}

Le Building Information Modeling (BIM) est un ensemble de technologies et méthodes de travail de plus en plus utilisées et plébiscitées à travers le monde. La France n'est pas précurseuse au niveau international dans ce domaine (Hochscheid, Halin, 2018c), pourtant, le BIM préoccupe beaucoup les professionnels du secteur de la construction et les architectes français. Si certains d'entre eux sont désireux de mettre en place des pratiques

\footnotetext{
* Corresponding author: elodie.h@crai.archi.fr
} 
BIM, d'autres, au contraire, y sont fortement opposés. Dans un projet BIM, les architectes sont souvent les premiers acteurs à produire une maquette numérique; ils sont donc au cœur du processus de diffusion du BIM dans le secteur de la construction. Cependant, peu d'études se sont attachées à mesurer leur niveau d'adoption du BIM.

L'objet du travail présenté ici est d'identifier l'avancement moyen des agences d'architecture dans leur adoption du BIM, et les caractéristiques des agences qui mettent en œuvre ces pratiques aujourd'hui. Dans une première partie (titre ${ }^{\circ} 2$ ), nous présentons l'état des connaissances sur l'adoption du BIM par les agences d'architecture et la manière dont ces connaissances et leurs lacunes ont déterminé notre méthode de travail pour l'élaboration et la diffusion d'un questionnaire. Nous restituons ensuite les résultats de notre enquête : la seconde partie concerne l'avancement moyen des agences d'architecture dans l'adoption du BIM; la troisième évoque les liens identifiés entre le niveau d'utilisation du BIM et certaines caractéristiques démographiques des agences.

\section{Cadre de l'étude et méthode}

\subsection{Les enquêtes sur le BIM et les agences d'architecture}

Les enquêtes sur le BIM se sont multipliées ces dernières années : plusieurs études internationales sont publiées régulièrement sur l'adoption du BIM. C'est le cas notamment des études de SmartMarket (McGraw Hill Construction, 2010) et NBS (Waterhouse, 2019). Malgré l'abondance de données dans ces études, c'est souvent l'ensemble du secteur qui est étudié (pas des professions spécifiques) et les architectes y sont peu représentés.

En France, un premier baromètre (Groupe BATIACTU, 2018) a permis de dresser un panorama du BIM dans le secteur français, avec une étude reconduite trois années de suite pour observer les évolutions. Les chiffres y sont parfois présentés par corps d'état, rarement par profession. Les architectes sont donc souvent intégrés aux chiffres de la maîtrise d'œuvre. L'étude française récente d'Autodesk sur le BIM (Autodesk, 2019) implique quant à elle une proportion importante d'architectes dans son panel.

Le Conseil National de l'Ordre des Architectes (CNOA) publie régulièrement des chiffres sur la profession d'architecte dans "Archigraphie» (CNOA, 2018). La version 2018 du rapport ne comporte en revanche aucune occurrence du terme « BIM ».

Les enquêtes sur l'adoption du BIM reposent rarement sur les théories de diffusion et d'adoption des innovations. Elles ne permettent pas de faire le point sur les agences d'architecture, acteurs pourtant essentiels de la transition BIM. Les études sur la profession d'architecte fournissent quant à elles de nombreuses données sur les agences mais concernent peu leurs pratiques numériques et collaboratives. Les données disponibles sur l'utilisation du BIM par les architectes sont donc aujourd'hui lacunaires.

\subsection{Contexte de l'étude}

Les objectifs de l'enquête complète sont d'étudier (1) la démographie des agences d'architecture, (2) l'état des pratiques et l'appréciation du BIM dans les agences, et (3) les facteurs qui influencent la décision de commencer à travailler en BIM et qui en facilitent la mise en œuvre. Seule une partie de l'aspect 2 est ici présentée : le niveau d'adoption du BIM dans les agences et les caractéristiques de celles qui le mettent en œuvre.

L'enquête par questionnaire fait suite à des travaux préliminaires d'état de l'art, d'entretiens, de recherche-action et d'observation directe au sein d'agences d'architecture (Hochscheid, Halin, 2018b ; 2018c). Les méthodes qualitatives permettent en effet une première approche de l'objet d'étude (Kaufmann, 2011), et sont utiles pour problématiser le 
sujet (De Singly, 2016). Ce travail préliminaire nous a permis d'élaborer des hypothèses utiles à l'élaboration du questionnaire. Nous avons notamment travaillé à l'explicitation du processus d'adoption du BIM à partir de différentes théories de diffusion de l'innovation (Hochscheid, Halin, 2018a), ainsi qu'au référencement des facteurs qui peuvent influencer l'adoption du BIM (Hochscheid, Halin, 2019). Ces modèles ont servi de base à la construction du questionnaire dont une partie des résultats est présentée dans cet article.

\subsection{Protocole et mode de diffusion}

L'enquête comporte trois grandes parties: les caractéristiques des individus et agences répondants, les pratiques numériques au sein des agences, et les pratiques d'implémentation du BIM. Le questionnaire a été saisi dans LimeSurvey, un outil professionnel d'enquête en ligne. Une première version de celui-ci a été administrée à un panel de 6 personnes afin de vérifier l'intelligibilité des questions avant la diffusion. Cela a permis de corriger des points de syntaxe et de vocabulaire dans les questions. Un lien internet a ensuite été créé et diffusé par plusieurs moyens numériques. Le CNOA l'a diffusé début avril 2019 aux 30000 architectes inscrits dans leur base de données, en deux vagues de mail espacées d'une semaine. Ces modes de diffusion ont permis de recueillir 894 réponses complètes (questionnaire terminé envoyé par le répondant). Les réponses incomplètes ont été écartées car elles auraient introduit un biais dans l'analyse.

La représentativité du panel a été vérifiée en comparant des caractéristiques des répondants (origine géographique et taille des agences) aux statistiques officielles du CNOA présentées dans Archigraphie 2018. Le panel est identique aux chiffres officiels sur la répartition géographique des agences mais compte 10 points de moins d'agences de 1 à 5 personnes. Ceux et celles qui se sentent peu concernés par la thématique BIM ont pu être rebutés par le titre de l'enquête. Mais l'écart avec les chiffres du CNOA reste faible et le nombre de réponses est conséquent : la représentativité du panel est solide.

\section{L'adoption des pratiques BIM dans les agences}

Cette partie est un état des lieux général de l'adoption des pratiques BIM dans les agences. Les logiciels utilisés par ces dernières ainsi que leurs pratiques de conception assistée par ordinateur ont été étudiés pour en déduire leur «niveau de maturité BIM».

\subsection{Les logiciels utilisés par les architectes}

Nous avons demandé aux agences d'indiquer les logiciels qu'elles utilisent pour produire des documents graphiques pendant les phases de conception (Figure 1). Les répondants pouvaient cocher plusieurs modalités, car la phase d'entretiens a révélé que de nombreuses agences utilisaient plusieurs logiciels permettant d'effectuer des tâches similaires (Vectorworks et ArchiCAD par exemple).

$48 \%$ des répondants indiquent utiliser AutoCAD, et $46 \%$ SketchUp ; les deux logiciels étant souvent utilisés de manière combinée. 34\% des répondants utilisent ArchiCAD, 27\% Revit et seulement 5.3\% utilisent Allplan. Les répondants qui ont coché "Autre » ont indiqué des outils de rendu ou les logiciels Draftsight, PowerCADD, ou Arc+.

Tout d'abord, on note qu'une majorité d'agences travaille avec AutoCAD. Les agences utilisant des outils «BIM de conception » sont relativement nombreuses, et l'écart entre Revit et ArchiCAD est assez faible. ArchiCAD, logiciel du fournisseur européen Nemetschek, est historiquement plus présent en France que Revit. Il est en revanche tout à fait possible que les agences qui sont passées récemment sur un «outil BIM » se soient 
tournées vers Revit, restant alors dans l'environnement Autodesk en gardant une interface utilisateur similaire à ce qui était probablement leur précédent outil : AutoCAD. Pendant sa transition BIM, la France est également influencée par les standards internationaux qui ont largement généralisé les solutions logicielles de la suite Autodesk, dont Revit.

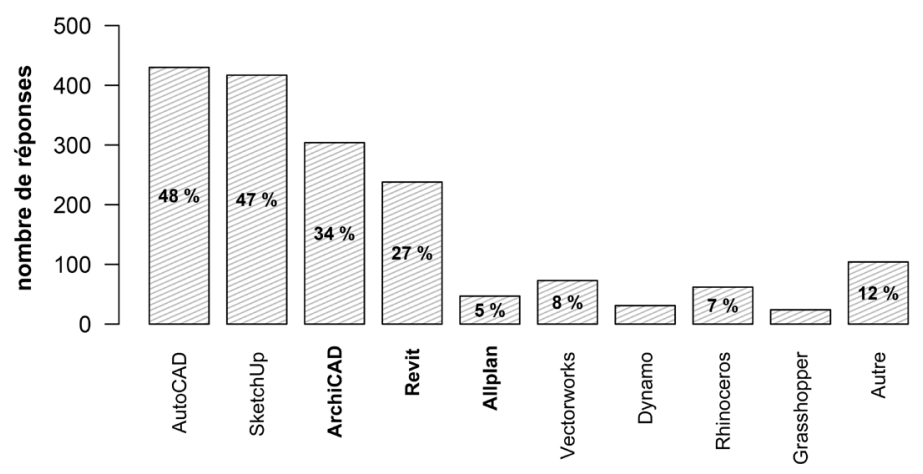

Figure 1. Logiciels de Conception Assistée par Ordinateur utilisés par les agences

Un tiers des répondants (293 personnes) ont coché plusieurs logiciels permettant d'effectuer des tâches identiques. Parmi eux, et ayant eu la possibilité de cocher plusieurs modalités de réponse, certains indiquent que les outils ne sont pas partagés par tous à l'agence parce que le choix des outils se fait sur préférence personnelle (44\%) ou selon les besoins dans le projet $(40 \%)$, parce qu'ils commencent à mettre en place des pratiques BIM $(15 \%)$ ou qu'ils les expérimentent pour voir si elles conviennent $(15 \%)$, qu'ils sont en fin de transition BIM (38\%), ou que certains sont revenus aux outils pré-transition BIM (7\%).

\subsection{Le niveau d'utilisation du BIM dans les agences d'architecture}

Nous avons demandé aux répondants de positionner leur agence par rapport à différentes pratiques BIM. Les réponses ont été exploitées pour positionner les agences françaises sur le processus d'adoption du BIM.

\subsubsection{Méthode}

Plusieurs types d'études ont vocation à déterminer le niveau d'utilisation du BIM des professionnels. A l'échelle macro, les baromètres dressent un panorama général de la diffusion du BIM dans le secteur de la construction; à l'échelle micro, les matrices de maturité évaluent la maturité BIM d'une entreprise voire d'un individu. L'objet de cet article est de déterminer l'état actuel de l'adoption et appréhension du BIM par les agences d'architecture : il s'agit d'un baromètre. Nous avons cependant vu (partie 2.1) que ce type d'étude manquait généralement de cadre théorique et de rigueur. Les matrices de maturité qualifient quant à elles la capacité d'une entreprise à mettre en œuvre des pratiques BIM (Succar, 2009 ; Absil et al., 2015). Elles n'évaluent ni son appréhension du BIM, ni son intention ou sa difficulté à le mettre en œuvre, qui sont des concepts centraux dans l'étude de l'adoption du BIM (Hochscheid, Halin, 2018a). De plus, elles évaluent cette capacité avec un degré de granularité très élevé, difficile à mettre en œuvre et non indispensable dans une enquête par questionnaire à grande échelle. Elles ne sont donc adaptées ni pour déterminer l'avancement de l'adoption du BIM des acteurs d'un secteur, ni pour déterminer leur niveau de maturité dans notre cas.

Dans le cadre d'une enquête par questionnaire, il est nécessaire de réduire le nombre de questions pour éviter l'abandon. Les niveaux de maturité sont donc étudiés ici avec un 
niveau de granularité faible. Ce sont les niveaux de maturité BIM (NdM) tels que définis par (Boton, Kubicki, 2014 ; Succar, 2009) qui sont ici utilisés. Ils qualifient les pratiques des acteurs en trois paliers. Le niveau 0 correspond au dessin assisté par ordinateur; le niveau 1 à la modélisation orientée objet sans échange de maquette numérique avec les partenaires; le niveau 2 correspond à une collaboration orientée modèle ; et le niveau 3 à l'utilisation collaborative synchrone d'un modèle stocké en réseau (Boton, Kubicki, 2014).

Nous avons demandé aux répondants de positionner leur agence par rapport à huit pratiques caractéristiques et révélatrices des niveaux 1 et 2 (Tableau 1). Le niveau 3 tel que décrit par (Boton, Kubicki, 2014) n'a pas été testé car les entretiens préliminaires l'ont révélé marginal; et le niveau 0 a été déduit de l'absence des niveaux 1 et 2.

Tableau 1. Huit pratiques caractéristiques des niveaux 1 et 2 testées dans notre enquête.

\begin{tabular}{|c|c|c|}
\hline Pratique & Description & Niveau \\
\hline P1 & Produire les plans et coupes depuis la 3D d'un logiciel BIM & 1 \\
\hline P2 & Réaliser des métrés (semi-)automatisés depuis la 3D & 1 \\
\hline P3 & Utiliser un serveur de travail collaboratif BIM interne à l'agence & 1 \\
\hline P4 & Renseigner des informations non géométriques dans le modèle 3D & 1 \\
\hline P5 & Transmettre des maquettes numériques aux BETs (en IFC) & 2 \\
\hline P6 & Importer/exploiter une maquette numérique reçue des BETs & 2 \\
\hline P7 & Formaliser les échanges de fichiers avec des partenaires & 2 \\
\hline P8 & Utiliser une plateforme collaborative BIM avec des partenaires & 2 \\
\hline
\end{tabular}

L'avancement des acteurs dans l'adoption du BIM est positionné sur le processus d'adoption du BIM (Hochscheid, Halin, 2018a) (élaboré à partir du modèle de Rogers (2003)). Pour chacune des huit pratiques, les répondants ont pu se positionner sur les différentes phases du processus d'adoption (Figure 2) en indiquant qu'ils ne savent pas de quoi il s'agit (phase 0), qu'ils savent de quoi il s'agit, sans plus (1), qu'ils pensent mettre en place un jour cette pratique à l'agence (2), qu'ils souhaitent la mettre en place très bientôt (3), qu'il est exclu pour eux de la mettre en place (3: gel), qu'ils la mettent en place actuellement (4), qu'ils ont essayé de la mettre en place mais que cela n'a pas fonctionné (4: gel), ou qu'ils ont généralisé cette pratique à l'agence (5).

\subsubsection{Résultats}

La figure 2 présente la répartition des agences françaises sur le processus d'adoption du BIM. Les niveaux de maturité 1 et 2 sont diffusés d'une manière très similaire dans la population d'agences (figure 2). Le niveau 1 est néanmoins plus souvent mis en œuvre (phase 5) que le niveau 2. Paradoxalement, les agences ayant indiqué « ne pas savoir de quoi il s'agit » (phase 0) au sujet des pratiques de niveau 1 sont plus nombreuses que pour le niveau 2. Ce résultat peut s'expliquer par le fait que le niveau 1 est maîtrisé depuis plus longtemps ; et le niveau 2, dont la mise en œuvre effective est plus récente, a été très plébiscité ces dernières années, probablement au détriment du niveau 1. Cela explique également bien les différences entre niveau 1 et 2 pour les phases 1,2 , et 3 . 
Le processus d'adoption peut se figer à différentes phases, notamment lors de la décision (3 : gel), ou de l'implémentation (4 : gel) (Hochscheid, Halin, 2018a). La proportion de répondants excluant la mise en œuvre de pratiques BIM (3: gel) est très importante, et concerne plus fortement le niveau 1 que le niveau 2 . Le nombre de répondants ayant tenté de mettre en œuvre des pratiques BIM sans y parvenir (4: gel) est quant à lui assez faible, et concerne légèrement plus le niveau 2 que le niveau 1.

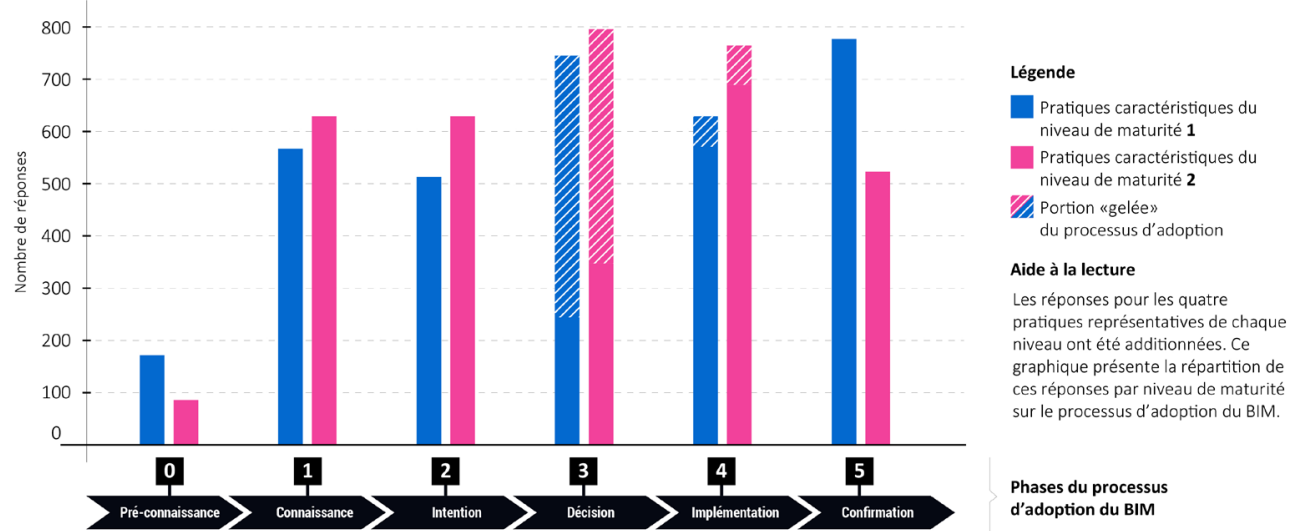

Figure 2. Répartition sur le processus d'adoption du BIM des réponses des agences pour les pratiques caractéristiques des niveaux de maturité 1 (P1-P4) et 2 (P5-P8).

Le NdM de chaque agence a été ensuite calculé sur ces mêmes réponses. Une agence est catégorisée de niveau 1 lorsqu'elle utilise un outil BIM et qu'elle a généralisé au moins une pratique caractéristique du niveau 1. Une agence est catégorisée de niveau 2 lorsqu'elle remplit les conditions du niveau 1 et a généralisé au moins deux pratiques de niveau 2. Les agences comptabilisées au niveau 1 ne sont pas au niveau 2. Celles qui ne sont ni au niveau $1 \mathrm{ni}$ au 2 sont comptabilisées en niveau 0 , ou inconnu si les données étaient manquantes.

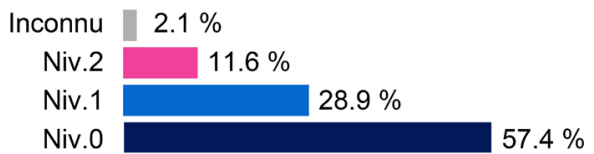

Figure 3. Niveau de maturité des agences d'architecture dans notre panel de répondants.

Le code couleur utilisé sur ce graphique est conservé sur les graphiques suivants.

D'après notre méthode de calcul, $57 \%$ des agences sont au niveau $0 ; 29 \%$ au niveau 1 , $12 \%$ au niveau 2, et $2 \%$ sont indéfinies (Figure 3 ). Ces chiffres sont très éloignés de ceux de l'étude d'Autodesk qui indique que $66 \%$ des répondants pratiquent le BIM.

La majorité des agences travaillent en niveau 0 . Les pratiques de niveau 1 semblent moins attractives et moins bien connues des agences qui ne les mettent pas en œuvre que celles de niveau 2. Les agences qui excluent l'implémentation de pratiques de niveau 1 sont plus nombreuses que pour les pratiques de niveau 2.

\section{Qui met en œuvre des pratiques BIM ?}

L'idée que « seules les grosses structures pourraient adopter la nouvelle technologie tellement son coût serait important » (Tripier, 2018) est très répandue. L'enquête a permis de croiser des informations sur la structure des agences avec leur NdM pour révéler que le BIM n'est pas implémenté seulement par ces « grosses structures». 
Une régression logistique multiple a été réalisée pour chaque NdM afin de caractériser les associations entre le niveau de maturité des agences (précédemment calculé) et certaines de leurs caractéristiques : le nombre de personnes à l'agence, le type et la taille des projets qu'elle réalise, le type de maîtrise d'ouvrage (publique/privée) avec qui elle travaille, son mode de collaboration avec les bureaux d'études techniques (BET), le renouvellement de personnel, le fonctionnement hiérarchique de l'agence, les modalités de collaboration en interne, la manière dont le travail est réparti, l'ancienneté du dirigeant et des employés, le niveau de participation du dirigeant à la production graphique, et l'intérêt du dirigeant/des employés pour les outils numériques. Les résultats les plus saillants sont présentés ici.

L'intérêt des employés pour le BIM est, dans notre jeu de données, la variable la plus liée au niveau de maturité des agences. Seuls $4 \%$ de celles qui ont des employés réticents ou indifférents au BIM sont au niveau 2 (Figure 4), 78\% au niveau 0. Mais la statistique ne dit pas si c'est l'intérêt des employés pour le BIM qui a poussé (ou freiné) la décision du dirigeant de passer l'agence en BIM ou si c'est la mise en place du BIM qui a rendu les employés réceptifs. La phase d'entretiens a cependant mis en évidence que la réticence des employés peut bloquer la mise en place du BIM à l'agence et que des employés proactifs peuvent au contraire convaincre le dirigeant d'engager son agence dans une transition BIM.

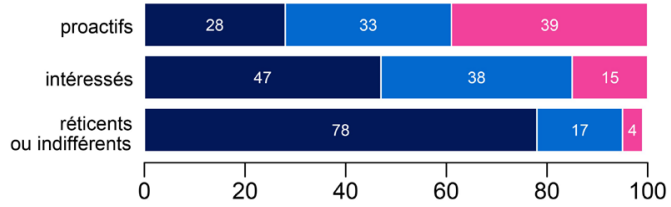

Figure 4. Proportions d'agences niveau 0,1 et 2 par rapport à la réaction des employés au BIM.

Le niveau de participation du dirigeant à la production graphique de l'agence est la seconde variable la plus fortement associée au NdM 2 dans une agence. Il est en effet cinq fois plus fréquent d'observer le niveau 2 dans les agences dans lesquelles le dirigeant participe à la production que dans celles dans lesquelles il n'utilise pas du tout les outils de production (Figure 5). Cette observation contredit par ailleurs une hypothèse élaborée après les entretiens: il était apparu que les dirigeants manipulant un peu les outils étaient réticents à en changer car, pour eux, cela marquerait leur éloignement avec la production graphique de l'agence. Le croisement entre le niveau de participation des dirigeants à la production graphique et leur perception de l'utilité du BIM (Figure 5) ne permet pas de conclure.
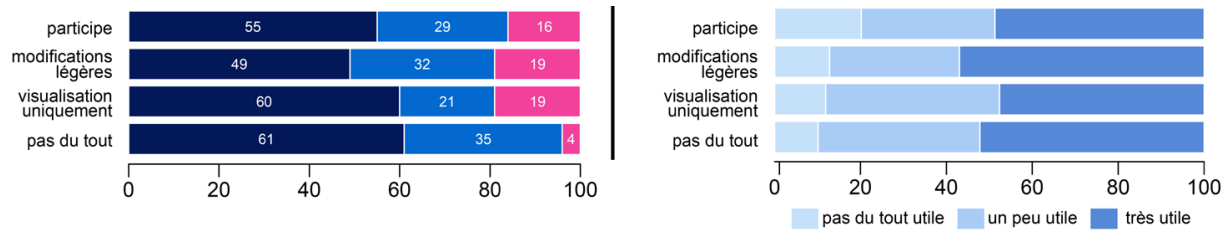

Figure 5. Niveau de maturité des agences par rapport au niveau d'utilisation du dirigeant des outils de CAO de l'agence (à gauche), et niveau d'utilité perçue du BIM du dirigeant par rapport à son niveau de participation à la production graphique de l'agence (à droite).

La taille des projets sur lesquels travaillent les agences est également liée à leur pratique du BIM. Plus il est fréquent qu'une agence réalise des projets de petite taille, moins il est probable qu'elle soit BIM niveau 2 (Figure 6). Mais passée une certaine échelle de projet (de grande taille), les agences sont moins fréquemment au niveau 2. 
Les répondants ont indiqué les types de programmes (Figure 6) sur lesquels l'agence travaille (avec la possibilité de cocher plusieurs réponses). Les agences qui réalisent des projets urbains sont pour un quart d'entre elles en BIM niveau 2. Cette proportion tombe à seulement $8 \%$ pour celles qui réalisent des maisons individuelles. Mais quel que soit le type de projet concerné, il y a toujours entre un quart et un tiers d'entre elles au niveau 1.

Le type de maîtrise d'ouvrage (MO) pour lequel travaillent les agences est lié au niveau $2: 7 \%$ des agences qui travaillent principalement en MO privée sont au niveau 2, contre $15 \%$ pour celles qui travaillent principalement en MO publique.
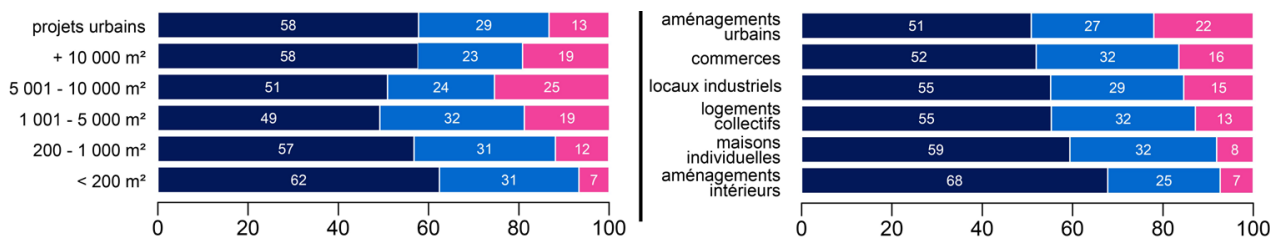

Figure 6. Niveau de maturité des agences par rapport à la taille moyenne de leurs projets (à gauche) et par rapport aux types de projets souvent réalisés (à droite).

La régression n'a pas révélé d'association forte entre la taille des agences (Figure 7) et leur niveau de maturité. Les indépendants travaillant seuls sont moins fréquemment au niveau 2 que les autres et les petites agences sont plus souvent au niveau 0 . Mais le pourcentage d'agences au niveau 2 est constant, qu'elles comptent 6 personnes ou plus de 20. Le niveau 1 est donc peu dépendant de la taille des agences, même s'il est un peu plus présent dans celles de 11 à 20 personnes que les autres. Plus marquant encore, les agences de plus de 20 personnes sont plus souvent en BIM 0 que les agences de 6 à 10 personnes. Si le coût du BIM est difficile à supporter pour les petites entreprises, les plus grandes font quant à elles face à une très forte inertie qui rend tout changement complexe.
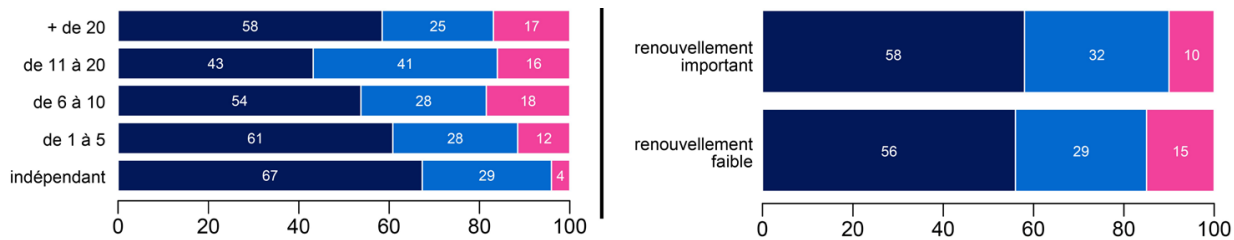

Figure 7. Niveau de maturité des agences par rapport au nombre de personnes qui y travaillent (à gauche), et au niveau de renouvellement du personnel ou « turnover » (à droite)

Une corrélation a été établie entre le type de hiérarchie dans les agences et leur niveau de maturité BIM : celles au système hiérarchique très vertical sont rarement BIM niveau 2. Les entretiens ont néanmoins montré que les agences à hiérarchie horizontale ont des difficultés à mettre en place des méthodes de travail communes. L'analyse par régression montre que les agences à fort renouvellement de personnel (Figure 7) sont moins fréquemment au niveau 2 que les autres. La phase d'entretiens a permis d'observer que le départ régulier d'employés empêchait l'ancrage de pratiques partagées au sein des agences et freinait la mise en place du BIM, qui nécessite une certaine stabilité.

\section{Discussion}

La méthode de calcul du niveau de maturité proposée et mise en œuvre dans cet article a un faible degré de granularité mais néanmoins ici suffisant. Des études préliminaires ont permis d'identifier que les agences adoptent le BIM en deux modes successifs : d'abord un changement radical qui fait basculer l'agence dans un autre niveau de maturité BIM, 
notamment lorsqu'elles changent d'outil principal de travail ou de méthode; puis incrémental, lorsqu'elles affinent leurs pratiques. Les 8 pratiques testées dans cette étude révèlent donc le palier de niveau de maturité des agences.

D'après la méthode de calcul ici utilisée, $29 \%$ des agences ont adopté le niveau de maturité 1 et $12 \%$ le niveau 2 . Les agences qui excluent l'intégration du niveau 1 en leur sein sont aujourd'hui plus nombreuses que celles qui souhaitent le mettre en place très bientôt. Le nombre d'agences en cours de transition vers le BIM ou souhaitant mettre en place des pratiques de niveau 1 ou 2 est cependant conséquent. Pour les agences qui ne les ont pas mises en œuvre, les pratiques de niveau 1 semblent moins attractives et moins connues que celles de niveau 2. La communication (commerciale, gouvernementale) réalisée autour du BIM se concentre sur le niveau 2, alors que le niveau 1 porte un potentiel très important pour les agences. Le niveau 1 suscite aussi plus de réticences que le niveau 2. Ce qui rebute les architectes par rapport au BIM concerne peutêtre plus le changement d'outil et de pratiques de dessin que l'échange avec des partenaires. D'ailleurs, les agences qui sont au niveau 1 sont généralement peu réticentes au niveau 2.

Le niveau de maturité BIM des agences est associé à certaines de leurs caractéristiques internes, surtout pour le niveau 2. En revanche, il y a toujours environ un quart à un tiers d'entre elles au niveau 1, quelle que soit la caractéristique d'agence testée. Celles qui sont avancées dans les pratiques BIM réalisent des projets plutôt moyensgrands et souvent en maîtrise d'ouvrage publique, avec un dirigeant qui manipule un peu les outils de production graphique. Le niveau de maturité 2 des agences n'est que faiblement lié à leur taille. On note même que les grandes agences sont plus souvent au niveau 0 (et aussi souvent au niveau 2) que les agences moyennes. Un fort turnover et un fonctionnement hiérarchique vertical sont associés à un faible avancement BIM. L'intérêt des employés pour le BIM semble être très moteur pour les agences : il est fréquent que des employés proactifs sur le BIM entraînent avec eux toute l'équipe. Or ces initiatives sont bloquées dans un système hiérarchique vertical où le dirigeant exclut un passage au BIM.

\section{Conclusion}

L'enquête par questionnaire ici présentée a permis de quantifier et qualifier le niveau d'adoption du BIM dans les agences d'architecture en France.

La majorité des agences ne travaillent pas en BIM, et peu d'agences sont au niveau 2. Elles sont nombreuses à indiquer que le BIM est complexe et trop coûteux. Environ un tiers d'entre elles travaille aujourd'hui avec un «outil BIM»; mais celles qui en exploitent pleinement le potentiel restent malgré tout rares. L'utilisation de ces outils et la mise en ouvre de pratiques de niveau 1 est, dans le jeu de données exploité, presque indépendant des caractéristiques des agences, et les agences qui comptent de nombreux collaborateurs n'ont pas des pratiques BIM plus avancées que les autres.

Les agences sont majoritairement favorables au BIM, mais ce dernier suscite également des réactions vives et négatives de la part des architectes. Celles-ci semblent plus liées au changement d'outil de dessin qu'aux échanges avec les partenaires du projet. Florent Champy notait, il y a presque vingt ans maintenant: "si l'intervention de l'architecte se justifie par sa capacité à élaborer des dessins [puisque l'identité de la profession s'est construite sur celle de l'architecte-artiste], la conception assistée par ordinateur, en simplifiant considérablement ce travail, recèle une menace. " (Champy, 2001). Il est possible que les craintes des architectes au sujet du niveau de maturité 1 soient issues du parcours historique de la profession et de son lien au dessin. Mais il faut souligner que de nombreuses agences s'approprient progressivement les pratiques BIM : l'impact de la trajectoire historique de la profession est donc également à nuancer. 


\section{Bibliographie}

Absil, Fabrice, De Boissieu, Aurélie, Daher, Elie, Ferriès, Bernard, Goulette, Jean-Pierre, Guerriero, Annie, Kubicki, Sylvain, Marques, Sandra et Reiter, Sandrine (2015). Méthode BIMetric, Grille 1. . 2015. Disponible à l'adresse : http://bimetric.list.lu/.

Autodesk (2019). Maîtrise d'oeuvre et maîtrise d'ouvrage en pleine révolution BIM - état des lieux, perceptions et axes de développement. 2019. Disponible à l'adresse: https://www.autodesk.fr/solutions/bim/explore-building-design/bim-study.

Boton, Conrad et Kubicki, Sylvain (2014). Maturité des pratiques BIM: Dimensions de modélisation, pratiques collaboratives et technologies. SCAN'14, 6ème Séminaire de Conception Architecturale Numérique. 2014. p. 45-56. Disponible à l'adresse: https://hal.archives-ouvertes.fr/hal-01025675/.

Champy, Florent (2001). Sociologie de l'architecture. Paris : La Decouverte. Repères, 314. ISBN 978-2-7071-3470-7.

CNOA, Conseil national de l'ordre des architectes (France) (2018). Archigraphie - une étude économique de la commande en architecture. 2018. Disponible à l'adresse: https://www.architectes.org/sites/default/files/atoms/files/archigraphie-3-web_0.pdf.

De Singly, François (2016). Le questionnaire. Paris : Armand Colin Paris.

Groupe BATIACTU (2018). Le BIM dans le secteur du BTP. 2018. Disponible à l'adresse : https://bit.ly/batiment-numerique-barometre2018.

Hochscheid, Elodie et Halin, Gilles (2018a). A model to approach BIM adoption process and possible BIM implementation failures. Creative Construction Conference. Ljubljana, Slovenia : 30 juin 2018. Disponible à l'adresse : www.bit.ly/EH_CCC2018.

Hochscheid, Elodie et Halin, Gilles (2018b). BIM implementation in architecture firms : Interviews,case studies and action research used to build a method that facilitates implementation of BIM processes and tools. Proceedings of the 36th eCAADe annual conference. Lodz, Poland: 2018. p. 10. Disponible à l'adresse : www.bit.ly/EH eCAADe2018.

Hochscheid, Elodie et Halin, Gilles (2018c). L'adoption du BIM dans les agences d'architecture en France. Actes du Séminaire Conception Architecture Numérique 2018. Nantes, France : octobre 2018. Disponible à l'adresse : www.bit.ly/EH_SCAN18.

Hochscheid, Elodie et Halin, Gilles (2019). A framework for studying the factors that influence the BIM adoption process. CIBw78. Newcastle, England : 18 septembre 2019. p. 275-285. Disponible à l'adresse : www.bit.ly/EH_CIB2019.

Kaufmann, Jean-Claude (2011). L'entretien compréhensif. Paris : Armand Colin.

McGraw Hill Construction (2010). The business value of BIM in Europe: Getting building information modelling to the bottom line the united kingdom, France and Germany. Smart Market Report. 2010.

Rogers, Everett M. (2003). Diffusion of Innovations, 5th Edition. Simon and Schuster. ISBN 978-0-7432-5823-4.

Succar, Bilal (2009). Building information modelling maturity matrix. Handbook of Research on Building Information Modeling and Construction Informatics: Concepts and Technologies, IGI Global. 2009. p. 65-103.

Tripier, Pierre (2018). L'architecture comme profession : comprendre les rapports de force. Profession Architecte: Identité - Responsabilité - Contrats - Règles - Agence - Economie - Chantier. Paris, France : Eyrolles. Profession. p. 69-72. ISBN 978-2-212-14380-5.

Waterhouse, Richard (2019). NBS International BIM Report. . 2019. Disponible à l'adresse : https://www.thenbs.com/knowledge/national-bim-report-2019. 